\title{
Peculiarities of Dielectric Relaxation in DMAAS Ferroelectric Crystals
}

\author{
V. KapustianiK ${ }^{a}$, Z. Czapla ${ }^{b}$ And R. Czukwinski ${ }^{b, c}$ \\ ${ }^{a}$ Ferroics Physics Laboratory, Physical Department, Lviv \\ I. Franko National University, Dragomanova st. 19, UA-79005 Lviv, Ukraine \\ ${ }^{b}$ Institute of Experimental Physics, University of Wrocław \\ pl. M. Borna 9, 50-204 Wrocław, Poland \\ ${ }^{e}$ Lower Silesian University College of Education \\ Wagonowa 9, 53-609 Wrocław, Poland
}

(Received February 15, 2002; revised version June 17, 2002)

\begin{abstract}
This paper presents the results of investigations of the dielectric properties of $\mathrm{NH}_{2}\left(\mathrm{CH}_{3}\right)_{2} \mathrm{Al}\left(\mathrm{SO}_{4}\right)_{2} \times 6 \mathrm{H}_{2} \mathrm{O}$ crystals performed in a wide frequency range. In particular, the temperature and frequency dependences of the dielectric permittivity were measured in the region of ferroelectric phase at different rates of temperature change. The thermal dipole relaxation with a critical slowing-down at $T_{\mathrm{c} 1}$ was observed at comparatively low frequencies of measuring field. The relaxation connected with the domain freezing takes place at still lower frequencies (below $100 \mathrm{kHz}$ ) and was found to be strongly dependent on the rate of the temperature change. It was shown that deuteration results in a noticeable change of the parameters characterising this process including the temperature of domain wall freezing.
\end{abstract}

PACS numbers: 64.70.-p, 77.84.-s

\section{Introduction}

Recently the crystals of dimethylammonium aluminium sulphate hexahydrate $\mathrm{NH}_{2}\left(\mathrm{CH}_{3}\right)_{2} \mathrm{Al}\left(\mathrm{SO}_{4}\right)_{2} \times 6 \mathrm{H}_{2} \mathrm{O}$ (DMAAS) were widely studied using different experimental methods. In particular, it has been found that DMAAS shows ferroelastic properties at room temperature and undergoes a proper ferroelectric phase transition of 2 nd order $2 / m \rightarrow m$ at $T_{c 1}=152 \mathrm{~K}[1,2]$. In works of [3-5] the anomalies of the dielectric and optical parameters have been found at $T_{\mathrm{c} 2}=110.5 \mathrm{~K}$ and $T_{1}=75 \mathrm{~K}$. The low temperature anomaly was related to the transition into the 
antiferroelectric phase, whereas the temperature region between $T_{c 2}$ and $T_{1}$ was characterised by a co-existence of ferroelectric and antiferroelectric phases [5]. Structural studies of DMAAS [6] have proved that orderings of DMA groups located in lattice voids are responsible for either of the phase transitions (PTs) while other changes in the lattice structure are just accompanying phenomena. $\mathrm{NH}_{2}$ group of DMA cation can occupy four equilibrium positions related in pairs by the inversion transformation forming a slightly deformed cross. The ordering appearing in the ferroelectric (antiferroelectric) phase was supposed to be of a mixed nature: ferroelectric ordering along the $X$-axis is accompanied by antiferroelectric one along the $Y$-axis and vice versa [5].

The thermal dipole relaxation with a critical slowing-down at $T_{\mathrm{c} 1}$ was observed in DMAAS at rather low frequencies for such a type of phenomena: in the region of $1-10 \mathrm{MHz}$. The relaxation connected with the domain freezing was observed at lower frequencies (at least, below $100 \mathrm{kHz}$ ) and was found to be more clearly seen in the deuterated crystals (DMAASD) [7]. Nevertheless, it was difficult to perform the detailed analysis of the supposed domain freezing in the hydrogenated samples, for the observed effect was very weak in the investigated frequency window at usual conditions of the experiment. For this reason it would be interesting to search how the relaxation process connected with domain walls freezing can be influenced by experimental conditions especially by varying the rate of temperature change. It is obvious to consider the corresponding dielectric process as a nonequilibrium one. That is why it is important to investigate also the time dependence of the dielectric parameters in the proper frequency region. Under such circumstances the detailed dielectric measurements of these crystals were performed in a wide frequency range of $10^{2}-10^{6} \mathrm{~Hz}$ at different rates of temperature change.

\section{Experimental}

Good quality samples of DMAAS were grown by slow evaporation of water from aqueous solution of dimethylammonium and aluminium sulphates with addition of sulphur acid.

Dielectric measurements were performed on rectangular crystalline plates perpendicular to the ferroelectric axis (the longitudinal component of the dielectric permittivity). After polishing, conducting silver paste electrodes were used. The complex dielectric constant was measured using Hewlett-Packard 4275 A multifrequencial LCR-meter. The applied AC measuring electric field was $E=10 \mathrm{~V} / \mathrm{cm}$.

\section{Results and discussion}

Let us consider the conditions when the domain contribution into the longitudinal dielectric permittivity would be maximal. Taking into account that any 
transformation of the domain structure is an inertial process, one can suppose that its configuration at a certain temperature would considerably depend on the rate of temperature change. At the low rates the domain structure could relax to the equilibrium state and contribution of the dipoles with an intermediate polarisation within the domain walls into the dielectric susceptibility will be minimal. On the other hand, with rising the temperature change rate the domain contribution should grow. At the same time, one can expect that the effect would be more pronounced at lower frequencies of the measuring electric fields. This prediction is confirmed by the temperature dependences of the real and imaginary part of dielectric permittivity (Fig. 1) obtained at higher velocities of the temperature change than in the case described in [7]. At such conditions of the experiment the deviation of $\varepsilon^{\prime}$ from the Curie-Weiss law becomes clearly seen in the region of $T<T_{\mathrm{c} 1}$. Moreover, at the rate of $2 \mathrm{~K} / \mathrm{min}$ one can observe the characteristic plateau (shown by arrows) which is limited by a sharp drop of $\varepsilon^{\prime}$ at lower temper-
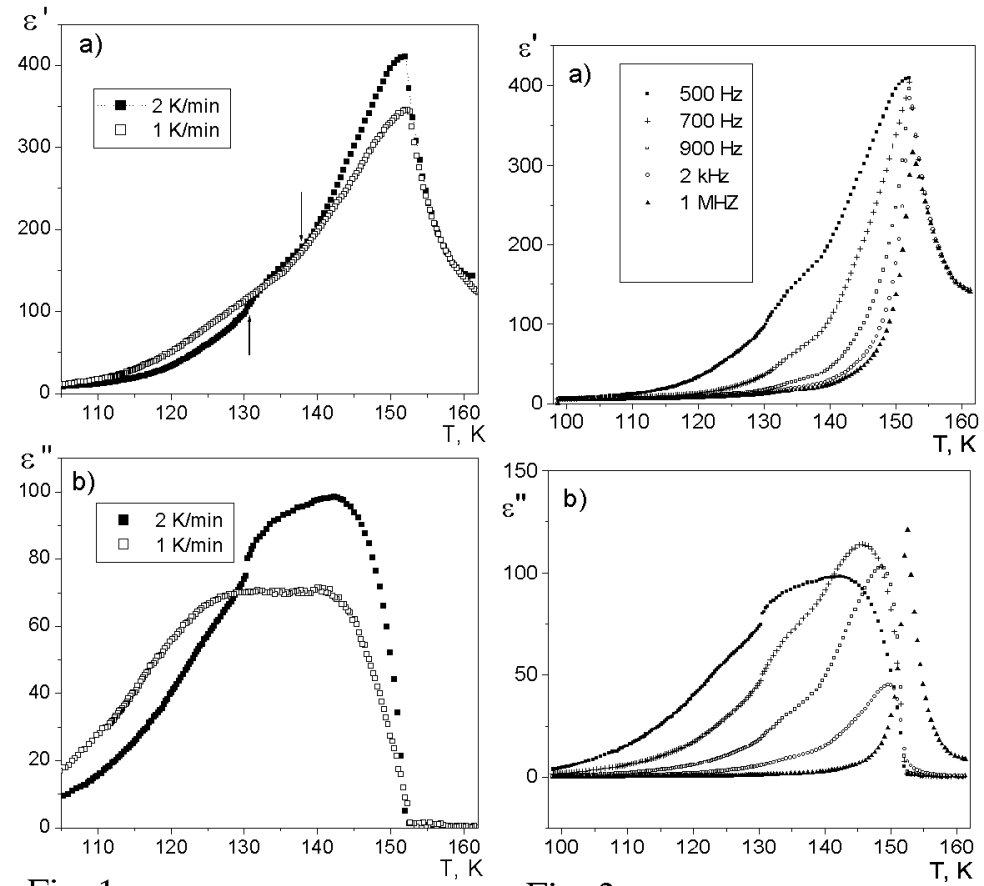

Fig. 1

Fig. 2

Fig. 1. Temperature dependences of the real $\varepsilon^{\prime}$ (a) and the imaginary $\varepsilon^{\prime \prime}$ (b) part of the dielectric constant obtained at different rates of cooling (frequency of measuring field $f=500 \mathrm{~Hz}$ ).

Fig. 2. Temperature dependences of the real $\varepsilon^{\prime}$ (a) and the imaginary $\varepsilon^{\prime \prime}$ (b) part of the dielectric constant of DMAAS obtained at the rate of cooling $2 \mathrm{~K} / \mathrm{min}$ at different frequencies of measuring field. 
atures. In the temperature dependence of $\varepsilon^{\prime \prime}$ the characteristic cup-like maxima appear. It is necessary to note that the higher the temperature change rate is, the sharper the anomalous changes of both dielectric parameters are. Such a behaviour of the dielectric permittivity (drop of $\varepsilon^{\prime}$ and corresponding maximum of $\varepsilon^{\prime \prime}$ ) corresponds to the temperature region of the freezing of the domain wall motion [7].

It is interesting to note that, contrary to the case of comparatively low rates of temperature change usually used in the dielectric measurements [7], at the rate of $2 \mathrm{~K} / \mathrm{min}$ the anomalies of $\varepsilon^{\prime}$ and $\varepsilon^{\prime \prime}$ reflecting the domain freezing process are observed also at the frequency of $1 \mathrm{kHz}$ (Fig. 2). The described effect also manifests itself in the frequency dependences of the dielectric parameters (Fig. 3). A sharp decrease in $\varepsilon^{\prime}$ and the corresponding maxima of $\varepsilon^{\prime \prime}$ are observed at the frequencies when the dipoles within the domain walls are no longer easily removable at the change of measuring field direction. At lower rates of temperature change the corresponding anomalies become smoother. That is why this effect was not observed
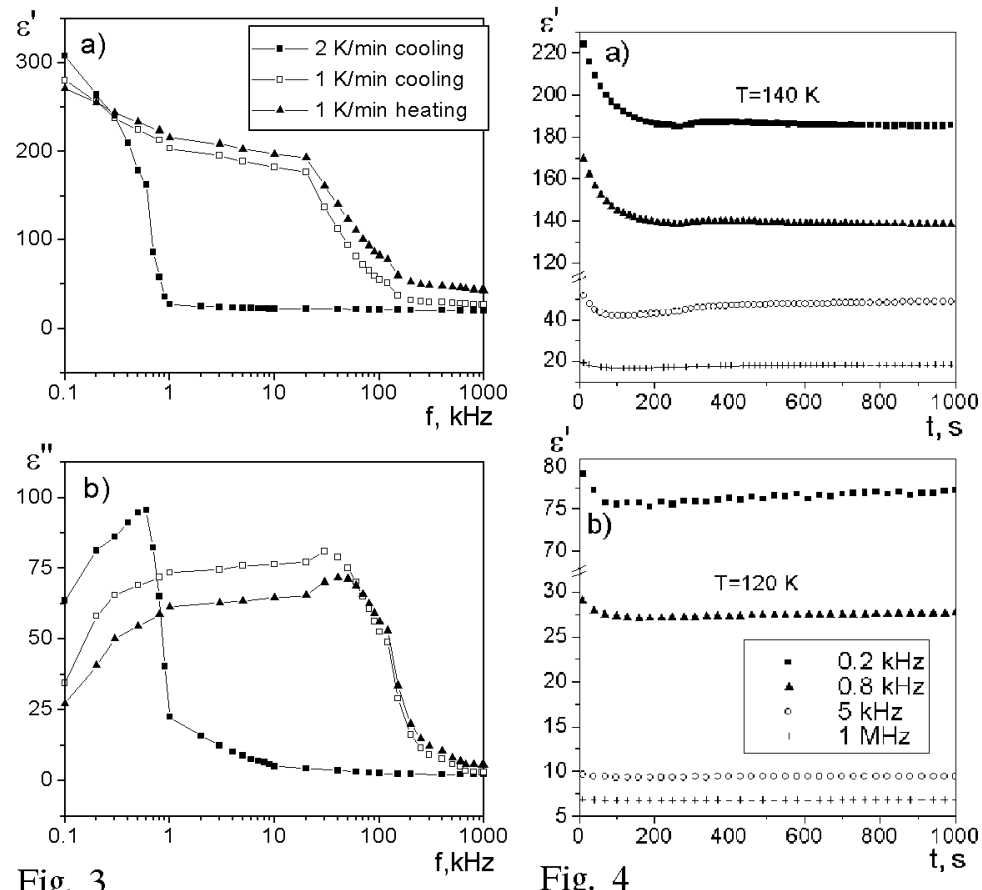

Fig. 3

Fig. 4

Fig. 3. The dielectric constants $\varepsilon^{\prime}(\mathrm{a})$ and $\varepsilon^{\prime \prime}$ (b) for DMAAS measured within the ferroelectric phase $(T=145 \mathrm{~K})$ as functions of frequency for various rates and directions of temperature change.

Fig. 4. Time evolution of the dielectric constant $\varepsilon^{\prime}$ for DMAAS obtained at different frequencies of measuring field within the ferroelectric phase: $T=140 \mathrm{~K}$ (a) and $T=120 \mathrm{~K}$ (b). 
at the usual conditions of experiment [7]. The analysis of the time dependence of the dielectric parameters (Fig. 4) confirms the aforementioned arguments. As it was expected, the temporal changes become the most noticeable at approaching the phase transition point at comparatively low frequencies of the measuring field. The characteristic time of the system transformation toward the equilibrium state (approximately $100 \mathrm{~s}$ ) determines the rate of temperature change (or the time of temperature stabilisation at every point of measurements) corresponding to the minimal contribution of the domain dynamics [7].

Finally, one can note that we have found the conditions of experiments (high rate of the temperature change and inessential widening of the working frequency range toward lower values) under which it is possible to observe in the Cole-Cole diagrams entirely the sector corresponding to the domain relaxation (Fig. 5).
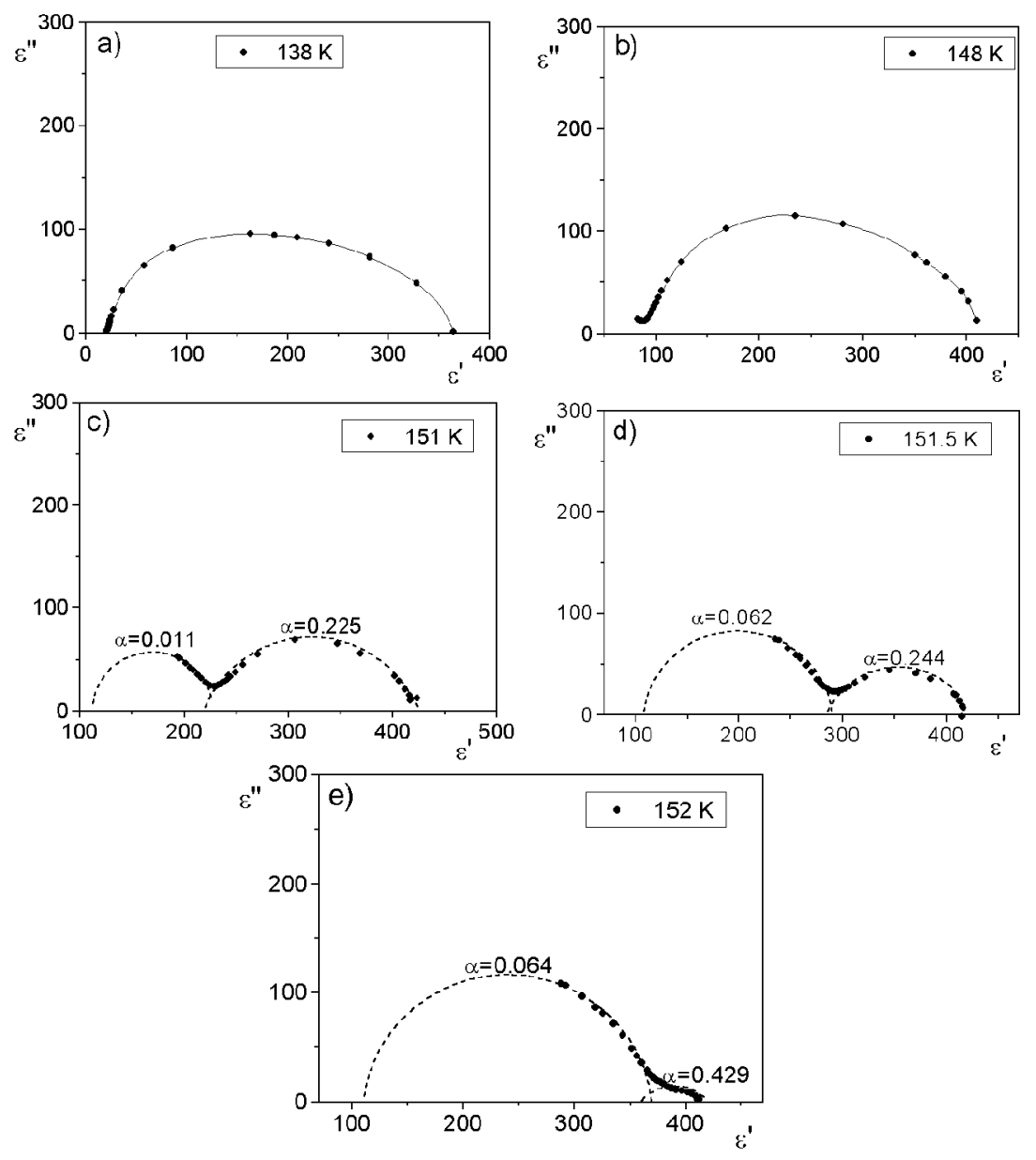

Fig. 5. Cole-Cole diagrams for DMAAS for different temperatures obtained at the rate of cooling $2 \mathrm{~K} / \mathrm{min}$. Solid lines (a,b) are the fits to Eq. (2). 
In particular, at $T=148$ and $151.5 \mathrm{~K}$, i.e. just below the PT point this part appears as a practically full semicircle which, however, is centred below the $X$-axis. This implies some distribution of relaxation time due to interaction between the dipoles [8]. A more detailed analysis of the relaxation process parameters for a certain temperature range within the ferroelectric phase is complicated due to the overlapping of the regions of domain and fundamental ferroelectric relaxations. The latter corresponds to the high frequency part of the Cole-Cole diagram (for $T=151.5 \mathrm{~K}$ as an example). Moreover, at temperatures $T>T_{\mathrm{c}}$ (beginning from $T=152.2 \mathrm{~K}$ ) only this part remains since in this case the domain contribution completely disappears.

One can note that the all presented diagrams deviate noticeably from the behaviour described by the Debye equation. Under such circumstances for the analysis of the high frequency part of the diagrams, as well as of the region of diagram overlapping, one can use the Cole-Cole equation with the empiric parameter $\alpha$. In this case the complex dielectric permittivity may be written as [8]

$$
\varepsilon^{*}(\omega)=\varepsilon_{\infty}+\frac{\varepsilon_{0}-\varepsilon_{\infty}}{1+(\mathrm{i} \omega \tau)^{1-\alpha}},
$$

where $\varepsilon_{\infty}$ stands for the high frequency limit, $\varepsilon_{0}$ is the permittivity obtained at static measurements, $\omega$ is the frequency of measuring field and $\tau$ is the relaxation time. The $\alpha$ parameter in Eq. (1) represents a measure of the relaxation time distribution.

For the analysis of the single slightly asymmetric low frequency parts of the diagrams (for $T=138 \mathrm{~K}$ and $148 \mathrm{~K}$ as an example) it would be convenient to use the model described in Ref. [7]. In this case the complex dielectric constant can be written as

$$
\varepsilon^{*}(\omega, T)=\left[\varepsilon_{0}(T)-\varepsilon_{\infty}(T)\right] \int_{0}^{\infty} \frac{g(\tau, T)}{1+\mathrm{i} \omega \tau} \frac{\mathrm{d} \tau}{\tau}+\varepsilon_{\infty}(T) .
$$

Here $g(\tau, T)$ results from a Gaussian distribution of activation energies $E$ centred around $E_{0}$ with the variance $\sigma$ which enters a Vogel-Fulcher law for the relaxation time [7]

$$
\tau(T)=\tau_{0} \exp \left(\frac{E}{T-T_{0}}\right),
$$

where $T_{0}$ is the Vogel-Fulcher temperature at which the relaxation time reaches infinity.

The Gaussian distribution of the activation energies $E$ is as follows:

$$
f(E)=\frac{1}{\sqrt{2 \pi \sigma}} \exp \left(\frac{-\left(E-E_{0}\right)^{2}}{2 \sigma^{2}}\right) .
$$

Then the function $g(\tau, T)$ takes the following form:

$$
g(\tau, T)=\frac{T-T_{0}}{\sqrt{2 \pi \sigma}} \exp \left(\frac{-\left[\left(T-T_{0}\right)\left(\ln \tau-\ln \tau_{0}\right)-E_{0}\right]^{2}}{2 \sigma^{2}}\right) .
$$


A choice of the temperature independent Gaussian distribution of the activation energy (5) looks natural for accounting of the fluctuations of the potential barrier magnitude $E$ from the average value $E_{0}[8]$.

While working out least-squares fits to the data (Fig. 5) and using a procedure described in detail elsewhere $[9,10]$ one can obtain the important parameters such as the temperature $T_{0}$, the characteristic relaxation time $\tau_{0}$, the mean value $\left(E_{0}\right)$, and the variance $(\sigma)$ for the distribution of activation energies

$$
\begin{aligned}
& T_{0}=116.0 \pm 0.1 \mathrm{~K}, \quad \tau_{0}=(5.8 \pm 0.2) \times 10^{-7} \mathrm{~s}, \\
& E_{0}=62.8 \pm 0.3 \mathrm{~K}, \quad \sigma=10.3 \pm 0.1 \mathrm{~K} .
\end{aligned}
$$

For comparison one can present the corresponding parameters obtained with the similar approximation of the Cole-Cole diagrams for the deuterated samples of DMAASD

$$
\begin{aligned}
& T_{0}=111.0 \pm 0.1 \mathrm{~K}, \quad \tau_{0}=(3.4 \pm 0.2) \times 10^{-7} \mathrm{~s}, \\
& E_{0}=77.7 \pm 0.3 \mathrm{~K}, \quad \sigma=16.0 \pm 0.1 \mathrm{~K} .
\end{aligned}
$$

One can note a certain difference between the parameters for the hydrogenated and deuterated crystals that reflects some dependence of the dipole reorientation process on the isotopic ratio of $\mathrm{H}$ atoms. First of all, the distribution of the activation energy becomes broader in DMAASD that should be expected at a partial deuteration of the sample.

On the other hand, it is interesting to note the correlation of the values $T_{0}$ with the temperature $T_{\mathrm{c} 2}$ of transition into the intermediate phase, obtained on the basis of electrooptic measurements and study of the transverse components of dielectric susceptibility [5]. This coincidence is connected with the analogy in the phenomena of domain wall freezing and the phase coexistence since both processes refer to the freezing of dipole orientations at a certain temperature (and/or with the influence of external electric field). Indeed, as it was supposed in [5] the phenomenon of phase coexistence in the DMAMeS $(\mathrm{Me}=\mathrm{Al}$, Ga) crystals should be ascribed to the "freezing" of dipoles in the position characteristic of the "overheated" low temperature phase or "supercooled" high temperature phase. Such a situation would be realized best of all at quick changes of the sample temperature. Otherwise, the reorientation of dipoles would proceed even at existence of high enough barriers between the position corresponding to the ferroelectric and antiferroelectric phases, and the region of phase co-existence would not be manifested by considerable anomalies of physical properties. From this point of view the presented experimental results correlate fairly well with the conclusions concerning the nature of the DMA groups ordering at low temperatures based on the data of previously performed study of the electrooptic effect and the transverse dielectric permittivity [5]. 


\section{Conclusion}

We have investigated DMAAS crystals around the ferroelectric phase transition by means of dielectric measurements over a wide frequency range. At the high rate of temperature change $(\sim 2 \mathrm{~K} / \mathrm{min})$ the thermal dipole relaxation with a critical slowing-down at $T_{\mathrm{c} 1}$ was observed as a clear semicircle in the Cole-Cole diagram at the frequencies rather low for such a type of phenomena. This allows us to confirm the conclusion $[5,7]$ that the flipping of heavy dimethylammonium groups between the two from the four possible stable orientations is responsible for the ferroelectric dispersion and that the ferroelectric phase transition is triggered by ordering these groups which carry the electric dipoles.

It has been also found that with increasing temperature change rate the domain contribution into the dielectric permittivity becomes more pronounced and corresponding dielectric dispersion is shifted toward low frequencies (at least, lower than $100 \mathrm{kHz}$ ). At such conditions of experiment it was possible to calculate the parameters of the dipole reorientation process which appeared to be clearly dependent on the isotopic ratio of $H$ atoms in a sample. In the same time, the Vogel-Fulcher temperature $T_{0}$ was found to be close to the temperature $T_{\mathrm{c} 2}$ of transitions into the intermediate phase [5] that reflects a similar nature of the domain wall freezing and the phase coexistence phenomena.

\section{References}

[1] L. Kirpichnikova, E. Andreyev, N. Ivanov, L. Shuvalov, V. Varikash, Kristallografiya 33, 1437 (1988).

[2] O. Vlokh, V. Kapustianik, I. Polovinko, S. Sveleba, V. Varikash, E. Andreyev, Zh. Prikl. Spektrosk. 51, 854 (1989).

[3] O.G. Vlokh, V.B. Kapustianik, I.I. Polovinko, E.F. Andreyev, V.M. Varikash, L.A. Shuvalov, Ferroelectrics 111, 333 (1990).

[4] V. Kapustianik, M. Bublyk, I. Polovinko, S. Sveleba, Z. Trybula, Phase Transit. 49, 231 (1994).

[5] V. Kapustianik, S. Sveleba, I. Stasyuk, O. Velychko, Z. Czapla, R. Tchukvinskyi, Phys. Status Solidi B 228, 785 (2001).

[6] A. Pietraszko, K. Lukaszewicz, L.F. Kirpichnikova, Polish J. Chem. 67, 1877 (1993).

[7] V. Kapustianik, M. Fally, H. Kabelka, H. Warhanek, J. Phys., Condens. Matter 9, 723 (1997).

[8] Yu. Poplavko, Physics of Dielectrics, Vyshcha shkola, Kyiv 1980.

[9] M. Fally, P. Kubinec, A. Fuith, H. Warhanek, C. Filipic, J. Phys., Condens. Matter 7, 2195 (1995)

[10] K. Kuramoto, J. Phys. Soc. Jpn. 56, 1859 (1987). 